\title{
The Deviation Analysis and Application of Correlation Tracking Algorithm
}

\author{
ZENG QINYONG ${ }^{1,2}$, HUANG ZHENGZHONG ${ }^{2}$, QIN KAIYU $^{1}$, and TANG XIANGCHENG ${ }^{2, \mathrm{a}}$ \\ ${ }^{1}$ University of Electronic Science and Technology of China, 61731 Chengdu, China \\ ${ }^{2}$ Southwest Institute of Technical Physics, 610041 Chengdu, China
}

\begin{abstract}
Correlation tracking algorithm is the most widely used image tracking algorithm . Its main error sources include processing error and positioning error. In order to improve the accuracy of correlation tracking algorithm, a mathematical model of positioning error based on the theory of random walk and martingale is set up, and the average time of positioning error exceeds the range is presented. The imageinterpolation method and surfaces-fitting method are put forward to suppress the positioning error and improve the accuracy._Simulation results show that: compared with the whole pixel tracking, quadratic interpolation tracking can double the accuracy, and quartic interpolation and surfaces-fitting can improve the tracking accuracy of about 4 times.
\end{abstract}

\section{Introduction}

On account of its simple structure, neither with the necessity of multiplication and division, nor needing comparison and estimation, Correlation Tracking Algorithm, possessed with highly real-time property, smoothly exerts the processor pipelining paralleling optimization and thus has won widespread application into engineering.

The essential idea of the related tracking is to identify and calculate in accordance with prestored targeted image templet: achieving traversal comparison between isometric areas of targeted templet and real-time image and gaining the related value of function, thereby figuring out the location of sub-image most similar to the targeted templet. As illustrated in Fig.1, when targeted image which is previously acquired becomes templet, traversal comparison can be realized in real- time image by similarity descriptor. Related values of function in respective locations compose related curved surface, the optimal point of which is exactly where the optimal location exists.

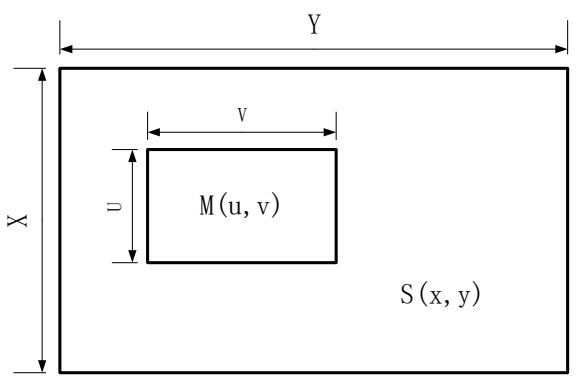

Figure 1. Traversal Matching of Templet in Image

$R(x, y)=\sum_{u=0}^{U} \sum_{V=0}^{V}|M(u, v)-S(x+u, y+v)|$
According to Fig. 1, the formula $\mathrm{M}(\mathrm{u}, \mathrm{v})$ represents templet, $\mathrm{S}(\mathrm{x}, \mathrm{y})$ demonstrates real-time searching area, $\mathrm{u} 、 \mathrm{v}$ show the locations of pixels in the templet, $\mathrm{U} 、 \mathrm{~V}$ indicate the size of templet, $\mathrm{x} 、 \mathrm{y}$ express the traversal location in searching area, and $\mathrm{R}(\mathrm{x}, \mathrm{y})$ reflects the value of location $(x, y)$ on related curved surface $R$.

As the capability of weapon equipment such as precision-guided weapon, airborne sighting pod and electronic counter equipment steadily facilitates, the precision of image tracker requires increasingly higher level. Restricted by the resolution of infrared image detector and operational capability of information processor, the hardware parameter of tracker can be hardly advanced dramatically. Therefore, it is of tangibly guiding significance for determining the deviation control strategy and improving tracking algorithm to summarize the types of deviation and establish deviation models, which will promote the arithmetic efficiency and tracking precision, facilitating the property of tracker's software.

This article will initially summarize and analyse the deviation of related tracking algorithm, putting forward certain feasible control strategy. After adopting multilevel interpolation and related surface fitting, the conclusion will finally be achieved.

\section{Analysis on Deviation of Related Tracking}

\subsection{Deviation Classification of Related Tracking}

In terms of tracking perspective, deviation should be classified into two types: process error and positional error.

\footnotetext{
a Corresponding author: txc_bs2010@163.com
} 
The process error is triggered under the condition that the actual matching point of each frame is the ideal one, when the posture of the target alters and the surface feature on the background differs, the real-time image will not accord with the referential model as the actual image is continuously changing, such as vehicles rising and falling, turning, leaning or being obscured by woods, and the image sequence will not be achieved by affine transformation from the same image.

In the single matching position, the known templet is not consistent with the actual image owing to some objective conditions and man-made interference; hence the actual location will be around the ideal one. The explanation is that the related tracking algorithm is based on gray level which can be obtained by pixel so that there should be some approximation describing on the gray level of non-integer pixel. Such circumstance will create positional error between used matching point and ideal one caused by common related algorithm only providing matching point of integer. Briefly speaking, the positional error is a kind of matching error that the optimal point is not the ideal one.

When process error and positional error are made in the related tracking, the reliability and accuracy of the whole tracking process will be negatively influenced.

\subsection{Premise on Deviation of Related Tracking}

Since the analysis of process error is complex involving various factors and a great deal of samples should be accumulated to make mathematical analysis, the article only summarizes the positional error.

When analyzing the positional error, process error is assumed to be zero, and process error and positional one is supposed to be mutually independent with same distribution. Due to the same property of direction $\mathrm{x}$ and direction $\mathrm{y}$, only direction $\mathrm{x}$ will be demonstrated in the analysis.

\subsection{Modeling of Positional Error}

\subsubsection{Accumulation of Positional Error}

Matching error will be made in the related matching of integer pixel. For example, the actual location of the target is 0.5 pixel whereas the optimal point should be integer 1 . If the primary location is 0 , the actual location is $\mathrm{Y}(\mathrm{t})$ at the time of $\mathrm{t}$, matching error is $\mathrm{X}(\mathrm{t})$, the optimal point at time 1 is $\mathrm{Z}(\mathrm{t})=\mathrm{Y}(\mathrm{t})+\mathrm{X}(\mathrm{t})$; if necessary, templet needs updating according to the optimal point matched in the former step, thus the considered actual location at time 2 has moved to location $\mathrm{Y}(2)+\mathrm{X}(1)$ ( the real actual location $\mathrm{Y}(1)$ has moved into $\mathrm{Y}(2))$. Therefore, the former matching error will be accumulated after updating the templet.

\subsubsection{Quantitative Analysis on Accumulated Positional Error}

Assuming Processor error is 0 , let $X_{n}$ be the error led by matching after updating templet at NO.n time, the accumulated error made by matching after updating templet n times should be $S_{n}=\sum_{j=1}^{n} X_{j}$

Assuming the error created by matching every time is independently-distributed random variable, and:

At the moment when matching point is within the certain range of actual location, matching error is considered to be 0 , which has $r$ of possibility of happening;

At the moment when matching point is beyond the certain range of actual location, matching error is considered to be positive, letting it be 1 , which has $\mathrm{p}$ of possibility of happening;

At the moment when matching point is less than the certain range actual location, matching error is considered to be negative, letting it be -1 , which has q of possibility of happening;

If the range interval is acquired small enough, modeling can be constructed by random differential equation; then the distribution of $X_{n}$ is like below:

$$
\begin{array}{r}
P\left(X_{n}=1\right)=p, P\left(X_{n}=0\right)=r, \\
P\left(X_{n}=-1\right)=q, \quad p+r+q=1 ; \quad(1)
\end{array}
$$

As supposed above, accumulated error $S_{n}$ is a random surfer model. Let the primary location of the target be 0 , a away from the left of the image, and $b$ away from the right of the image; then $S_{n}$ can be indicated as a particle randomly surfing in the state space $\{-a,-a+1, \ldots,-1,0,1, \ldots, b-1, b\}$.

Achieved by mathematical analysis:

Mean value of accumulated error:

$$
E\left(S_{n}\right)=n(p-q)
$$

Variance of accumulated error:

$$
D\left(S_{n}\right)=E\left(S_{n}^{2}\right)-\left(E S_{n}\right)^{2}=n\left[(p+q)-(p-q)^{2}\right]
$$

$T=\inf \left\{n: S_{n}=-a\right.$ 或 $\left.b\right\}$, then $\mathrm{T}$ is stopping time according to the definition of stopping time.

When $\mathrm{p}=\mathrm{q}$ and $S_{n}$ is a random surfer model, and both $\left\{S_{n}, n \geq 1\right\}$ and $\left\{S_{n}^{2}-n(p+q), n \geq 1\right\}$ are martingale, it can be achieved by Bounded Stopping Theorem that:

$$
\begin{aligned}
& E S_{T \wedge n}=0, \quad E S_{T \wedge n}^{2}=E[(T \wedge n)(p+q)] \quad(4) \\
& \text { Because }\left|S_{T \wedge n}\right| \leq \max (a, b): \\
& E[(T \wedge n)(p+q)]=E S_{T \wedge n}^{2} \leq \max \left(a^{2}, b^{2}\right)<\infty \quad \text { (5) } \\
& \text { Let } n \rightarrow \infty \text {,then } E T<\infty \text {.That is to say, }
\end{aligned}
$$
accumulated error will widen beyond the range of image within limited time.

Achieved by Wald Equation:

$$
E S_{T}=0, E S_{T}^{2}=(p+q) E T
$$


If the probability beyond the left borderline is recorded to be $P_{-a}=P\left(S_{T}=-a, T<\infty\right)$, and the probability beyond the right borderline $P_{b}=P\left(S_{T}=b, T<\infty\right)$, it can be achieved by $P(T<\infty)=1$ and $E S_{T}=0$ that:

$$
P_{-a}+P_{b}=1, \quad-a P_{-a}+b P_{b}=E S_{T}=0
$$

Thus: $P_{-a}=\frac{b}{a+b}, P_{b}=\frac{a}{a+b}$, and the average time made by accumulated error beyond the image range is:

$$
E T=\frac{E S_{T}^{2}}{p+q}=\frac{(-a)^{2} P_{-a}+b^{2} P_{b}}{p+q}=\frac{a b}{P+q}
$$

When $p \neq q$ and $S_{n}$ is a random surfer process, and both $\left\{S_{n}-n(p-q), n \geq 1\right\}$ and $\left\{\left(\frac{q}{p}\right)^{S_{n}}, n \geq 1\right\}$ are martingale, it can also be achieved by Bounded Stopping Theorem that:

$$
E S_{T \wedge n}=(p-q) E[T \wedge n], E\left(\frac{q}{p}\right)^{S_{T \wedge n}}=1
$$

While $\left|S_{T \wedge n}\right| \leq \max (a, b)$, then $E T<\infty$, and

$$
E T=\frac{E S_{T}}{p-q}<\infty
$$

I,e, accumulated error will expand beyond the image range within limited time.

Because $P(T<\infty)=1$ and $E\left(\frac{q}{p}\right)^{S_{T \wedge n}}=1$, let $n \rightarrow \infty$, then:

$$
P_{-a}+P_{b}=1, \quad\left(\frac{q}{p}\right)^{-a} P_{-a}+\left(\frac{q}{p}\right)^{b} P_{b}=1,
$$

Thus:

$$
P_{-a}=\frac{\left(\left(\frac{q}{p}\right)^{a}-\left(\frac{q}{p}\right)^{a+b}\right)}{1-\left(\frac{q}{p}\right)^{a+b}}, \quad P_{b}=\frac{\left(1-\left(\frac{q}{p}\right)^{a}\right)}{1-\left(\frac{q}{p}\right)^{a+b}},
$$

Average time made by accumulated error beyond the image range:

$$
E T=\frac{E S_{T}}{p-q}=\frac{b}{p-q}-\frac{a+b}{p-q} \frac{\left(\left(\frac{q}{p}\right)^{a}-\left(\frac{q}{p}\right)^{a+b}\right)}{1-\left(\frac{q}{p}\right)^{a+b}}
$$

\subsubsection{Analyzed Result of Accumulated Error Made by Positional Error}

When $\left\{X_{n}\right\}$ is discrete distribution whose value is only $-1,0$ or 1 , then:

- Accumulated error will increase beyond the image range within limited time;
- The average time of accumulated error increasing beyond the image range is...

$$
E T=\frac{b}{p-q}-\frac{a+b}{p-q} \frac{\left(\left(\frac{q}{p}\right)^{a}-\left(\frac{q}{p}\right)^{a+b}\right)}{1-\left(\frac{q}{p}\right)^{a+b}}
$$

\subsubsection{Method of Decreasing Error in Related Tracking}

As is proved by mathematical derivation above, the accumulation of positional error will increase beyond the image range within limited time, therefore, to decrease error in related tracking will turn into to decrease positional error, which can be considered as below:

- Interpolation Application

Indicated by previous derivation, interpolation will not have negative influence on distribution type of error. By continuously interpolating templet, positional error will gradually decrease to a certain lower bound.

- Related Surface Fitting Application

Theoretically, any continuous function can be infinitely approached by polynomial function, so fitting function similar enough to related curved surface created by specific texture must be found with massive experiments, by which positional error will infinitely approach zero. This kind of method, mutually independent on the former one, can be combined with interpolation together so that positional error will be effectively alleviated.

\section{Experiment and Analysis}

IR picture of outside test with resolution of $720 \times 576$ is selected, with vehicle image radiating from area $32 \times 32$ as templet, and the calculation within the searching range can be conducted, thereby the vehicle tracking test being completed as Fig. 2 . Finally the contrasts on tracking precision respectively between integer pixel tracking, twofold templet interpolation, fourfold templet interpolation and related surface fitting are achieved, as shown in Fig. 3 to Fig.6.

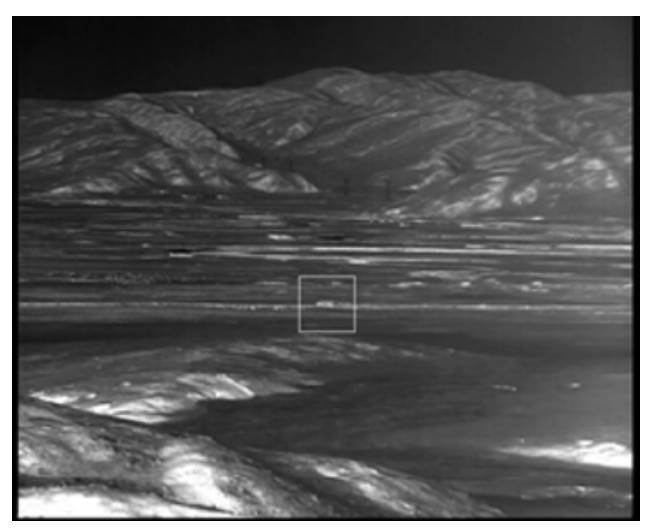

Figure 2. IR Picture of Vehicle Tracking Test.

The contrasts on tracking precision respectively between integer pixel tracking, twofold templet 
interpolation, fourfold templet interpolation and related surface fitting are achieved.

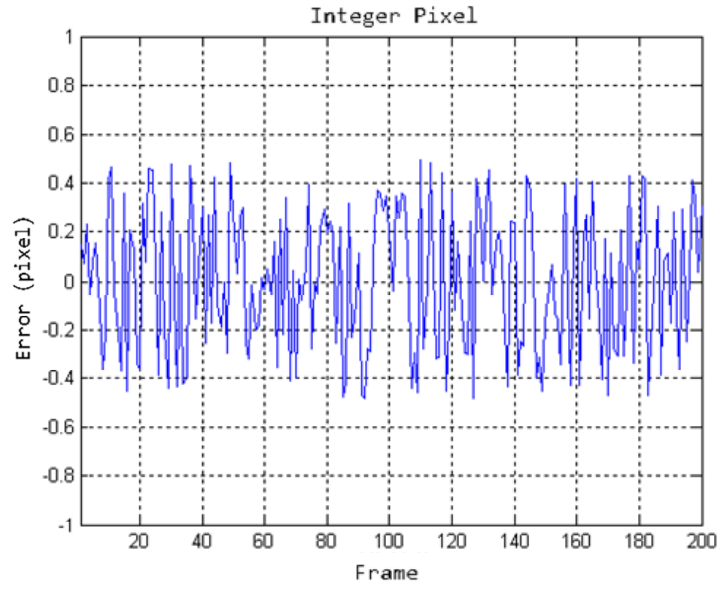

Figure 3. Integer Pixel of Image Interpolation.

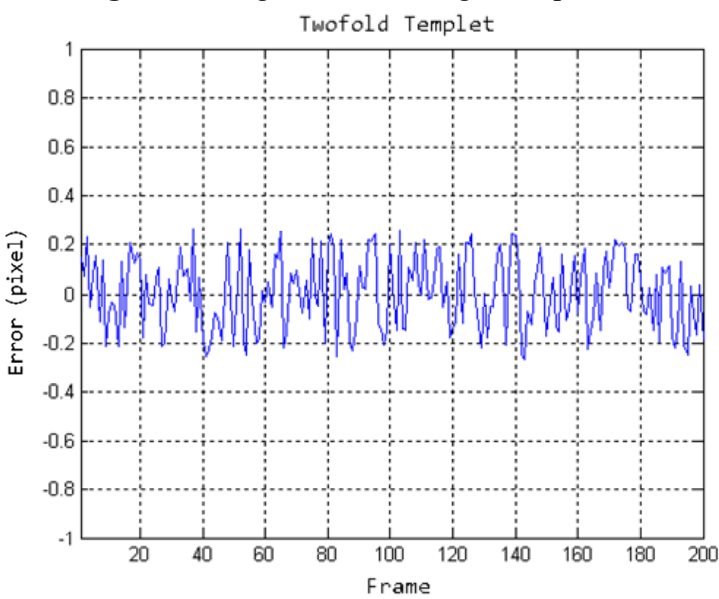

Figure 4 Twofold Templet of Image Interpolation.

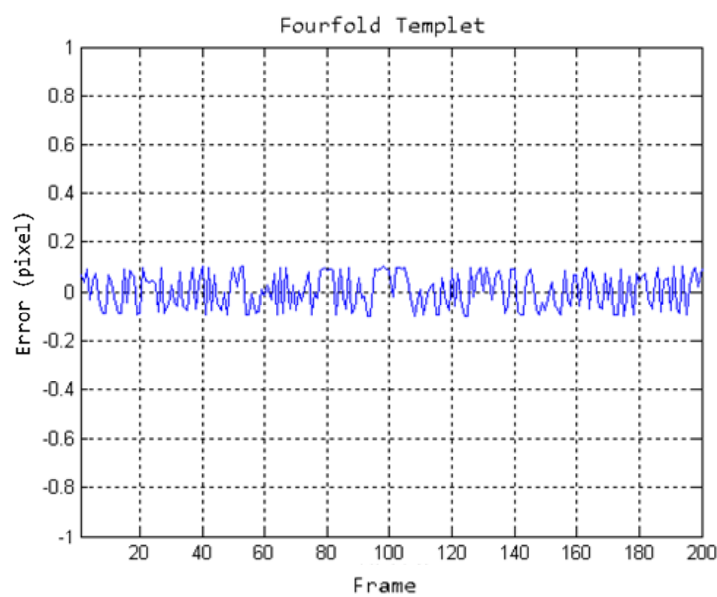

Figure 5. Fourfold Templet of Image Interpolation.

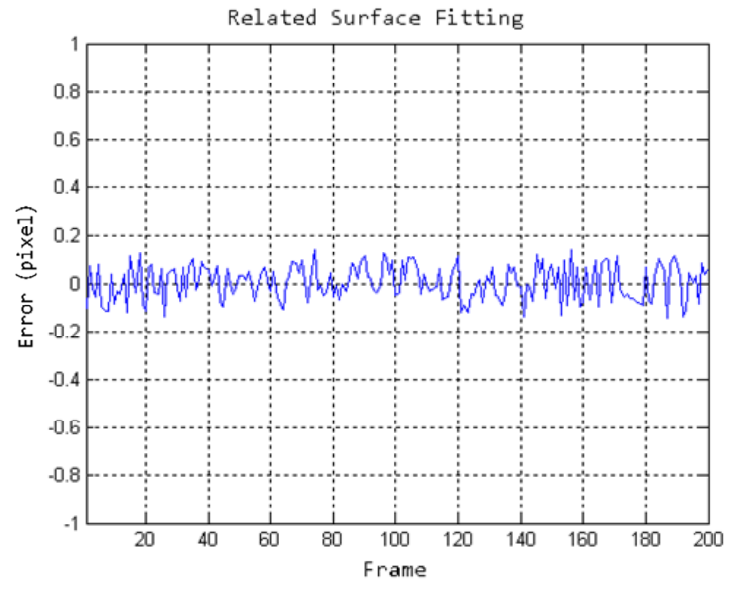

Figure 6. Related Surface Fitting.

As is summarized by the above analysis, integer pixel tracking error shows distribution within \pm 0.5 pixel; twofold templet interpolation most within \pm 0.2 pixel; fourfold templet interpolation and related surface fitting distribute mostly within \pm 0.1 pixel.

\section{Conclusion}

Related tracking error includes mainly process error and positional error. Assuming positional error $S_{n}$ is a random surfer model and a particle randomly surfing in the state space $\{-a,-a+1, \ldots,-1,0,1, \ldots, b-1, b\}$, mathematical analysis on the positional error of related tracking can be made based on the theory of random surfing and martingale, proving that positional error will increase beyond the image range within limited time and the average time increasing beyond is defined as ET(formula14). Image interpolation and related surface fitting, whose result examined by experiment analysis reveals higher level of interpolation matching higher tracking precision, are appropriate methods of decreasing tracking error. However, image interpolation drastically increases calculation, and the complication will interatively rise with the level growing. In the engineering practice, in the demand for breaking through the limitation of detector's resolution and information processor to motivate the tracking capability, accurate searching for the resources is a must, in which saved calculation resource can be applied into sub-pixel tracking founded on interpolation and related surface fitting, such as multilevel FOV guidance strategy, synthesized MEMS-IMU tracking strategy based on information integration, etc.

\section{References}

1. Matthias Mueller, Neil Smith, Bernard Ghanem. "Context-Aware Correlation Filter Tracking." CVPR (2017 oral)

2. A. Bibi and B. Ghanem. Multi-template scaleadaptive kernelized correlation filters. In IEEE International Conference on Computer Vision Workshops, ICCVW (2015) 
3. Y. Li and J. Zhu. A scale adaptive kernel correlation filter tracker with feature integration. In European Conference on Computer Vision Workshops, $\operatorname{ECCV}(2014)$

4. A. Bibi, M. Mueller, and B. Ghanem. Target Response Adaptation for Correlation Filter Tracking, pages 419-433. Springer International Publishing, Cham ( 2016)

5. Y Wang. Image Prepocessing Alogorithm Study on LUPA300. Changchun: Changchun University of Science and Technology(2011)

6. XiangC Tang. Highly-precise Image Tracking and Anti-interference Technology by Integration between Photoelectric Image and MEMS-IMU. Chengdu : China Research and Development Academy of Machinery Equipment( 2015) 